\title{
F-18-FDG and C-11-Choline Positron Emission Tomography in Human Esophago-Gastric Cancer: Prediction of Response to Therapy
}

\author{
Stuart Suttie ${ }^{\mathrm{a}, \mathrm{f}}$, Dympna McAteer ${ }^{\mathrm{b}}$, Margaret Sheehan ${ }^{\mathrm{c}}$, Marianne Nicolson ${ }^{\mathrm{d}}$, Lutz Schweigere, \\ Solveig Hammonds , Timothy Smith ${ }^{\mathrm{e}}$, Andrew Welch ${ }^{\mathrm{e}}$, Kenneth Park ${ }^{\mathrm{a}}$
}

\begin{abstract}
Background: To determine the utility of F-18-FDG and C-11-Choline uptake, in patients with esophageal and esophago-gastric junction tumors who are to undergo either neo-adjuvant or palliative chemotherapy, in predicting response (pathological and survival).

Methods: Eighteen patients with biopsy proven cancer were recruited prospectively. Patients underwent PET imaging before and during the first cycle of chemotherapy (seven and 14 days) with both F-18-FDG and C-11-Choline. Tracer uptake was quantified using Standardized Uptake Values. Pathological tumor response was determined using the Mandard criteria. Cellular proliferation was determined using ki-67 immunohistochemistry. Relationships between tracer uptake and response, one-year survival and cellular proliferation were determined.
\end{abstract}

Results: All 18 tumors were imaged by F-18-FDG PET compared to $16 / 18$ with $\mathrm{C}-11$-Choline. Change in uptake of either tracer did not correlate with pathological response. Pathological response did not influence survival (median-survival, responders $=16.1$ months; non-responders $=19.0$ months, $\mathrm{p}=0.978$ ). There was no significant correlation of change in tracer uptake with survival. C-11-Choline tumor uptake did not correlate with cellular proliferation.

Conclusions: F-18-FDG PET is superior for imaging of the primary tumor. Neither F-18-FDG nor C-11-Choline PET was able to predict response accurately.

Manuscript accepted for publication April 13, 2009

${ }^{\mathrm{a}}$ Department of Surgery, ${ }^{\mathrm{b}}$ Department of Radiology, ${ }^{\mathrm{c}}$ Department of Pathology, ${ }^{\mathrm{d}}$ Department of Oncology, Aberdeen Royal Infirmary, Foresterhill, Aberdeen, UK, AB25 2ZN

${ }^{\mathrm{e}}$ Department of Biomedical Physics, Aberdeen University, Aberdeen Royal Infirmary, Foresterhill, Aberdeen, UK, AB25 2ZN

${ }^{\mathrm{f}}$ Corresponding Author: Department of Surgery and Molecular Oncology, Ninewells Hospital and Medical School, Dundee, DD1 9SY

Email: sasuttie@hotmail.com

doi:10.4021/wjon2010.04.201w
Keywords: Positron emission tomography; Esophageal cancer; F18-FDG; C-11-Choline; Response

\section{Introduction}

Imaging modalities utilised in identifying response to therapy in gastro-esophageal cancer, include computerized tomography (CT) and endoscopic ultrasound (EUS), detecting anatomical changes within the tumor. These changes take several months to become apparent and may be indistinguishable from surrounding tissue edema. Positron Emission Tomography (PET) utilises metabolically active tracers to probe metabolic changes associated with response that precede morphological changes [1, 2]. Esophageal cancer has a poor prognosis [3], worse in those having no response to neo-adjuvant chemotherapy than patients proceeding directly to surgery. PET has potential benefits, theoretically allowing decisions regarding adjustments in chemotherapy to be made during the course of treatment.

[18F]2-fluoro-2-deoxy-d-glucose (F-18-FDG) is the most commonly used tracer for PET imaging and has been successful at imaging [4] and identifying response to therapy [5-7] in gastro-esophageal malignancies.

Carbon-11-Choline (C-11-Choline) PET has not been used to monitor response to chemotherapy in patients with gastro-esophageal cancers to date. Choline is a precursor for the synthesis of phospholipids and is required for transmembrane signalling and lipid-cholesterol transport $[8,9]$. Carcinogenesis and tumor cell growth are characterized by increased cellular proliferation and increased cell membrane synthesis. C-11-Choline uptake is thought to parallel cell proliferation, hence its theoretical use in predicting response.

The primary aim of our study was to determine if C11-Choline was superior to F-18-FDG PET for predicting pathological response and survival, in patients with esophageal and esophago-gastric junction tumors undergoing either neo-adjuvant or palliative chemotherapy. The secondary aim was to determine if C-11-Choline uptake correlated with cellular proliferation. 


\section{Patients and Methods}

\section{Patients}

The Grampian Research Ethics Committee approved the study. Eighteen patients with biopsy proven esophageal/ esophago-gastric junction (OGJ) cancers undergoing neoadjuvant or palliative chemotherapy were prospectively recruited. Before chemotherapy, all patients underwent staging CT. A subset of patients underwent EUS as part of the COGNATE trial [10]. Restaging following initial therapy was performed at the discretion of the primary surgeon/ oncologist, based on initial staging and clinical response to chemotherapy.

Patient inclusion criteria:1) Biopsy proven esophageal cancer (adenocarcinoma/squamous cell carcinoma); 2) Biopsy proven OGJ cancer (Siewert type I + II) [11]; 3) Eligible to receive neo-adjuvant/palliative chemotherapy $[12$, 13].

Patient exclusion criteria: 1) Diabetes mellitus

Patients who had histo-pathology and stage discussed at the weekly Multi-Disciplinary-Team-meeting, and judged suitable for curative surgery, underwent neoadjuvant chemotherapy prior to their surgery. Early in the study this incorporated cisplatin and 5-fluorouracil as per OE02 trial [12]. Latterly 5-fluorouracil was substituted with capecitabine (1250 $\mathrm{mg} / \mathrm{m}^{2}$ daily).

A subset of patients who had unresectable locally advanced disease at staging, but with adequate response to therapy, may become candidates for surgery had three cycles of triple combination therapy (epirubicin, cisplatin and capecitabine [13]) prior to restaging with CT. In patients with significant cardiac disease, epirubicin was substituted with mitomycin-C (60 mg/m², alternate cycles). Upon restaging, if disease progression was identified and the tumor deemed unresectable the patient underwent a course of concurrent chemoradiotherapy over five weeks (radiotherapy: $50 \mathrm{~Gy}$, 25 daily fractions for five weeks; chemotherapy: 33 days of capecitabine in $1300 \mathrm{mg} / \mathrm{m}^{2} /$ day with cisplatin in $20 \mathrm{mg} / \mathrm{m}^{2}$ weekly for five weeks).

Patients with advanced disease entered into the REAL2 study [13]. Patients with significant cardiac disease had epirubicin substituted with mitomycin-C (off-trial), and received three cycles then re-staged with CT. If there was a radiological response a further three cycles were given.

Baseline PET scanning was performed in the two week interval prior to commencing chemotherapy, then at day seven and 14 from the onset of first cycle of chemotherapy, using both F-18-FDG and C-11-Choline.

\section{PET protocol}

PET scans were performed on a CTI/Siemens ECAT-EXACT-31-scanner (Knoxville, Tennessee, USA). Patients were fasted for 6 hours prior to scanning to reduce circulating glucose levels and enhance F-18-FDG uptake. Blood glucose levels were measured prior to scanning. Patients Body Surface Area (BSA) were calculated according to the formula: BSA $\left(\mathrm{m}^{2}\right)=0.20247 \times$ Height $(\mathrm{cm})^{0.725} \mathrm{xWeight}(\mathrm{kg})^{0.425}$ [14] . Tumor site was marked on the anterior chest wall using information from CT and endoscopy, allowing a $10 \mathrm{~cm}$ window to be identified around the tumor. Subsequent PET scans were based on this one bed, in 2D acquisition mode, with transmission scans utilising a Gadolinium source. Images were reconstructed using filtered back projection with a Hanning window (cut-off at the Nyquist-frequency) and calibrated by imaging a cylindrical phantom of F-18-FDG on the same day as each image. F-18-FDG and C-11-Choline were manufactured on site. C-11-Choline studies were performed prior to the F-18-FDG due to Carbon-11s shorter half life.

\section{C-11-Choline}

A 10-minute transmission scan was acquired for attenuation correction over the $10 \mathrm{~cm}$ bed previously identified. All patients received an intravenous bolus of C-11-Choline (375 MBq; mean $318 \mathrm{MBq}$, range 198 - $396 \mathrm{MBq}$ ) after which imaging was immediately commenced for 40 minutes for a dynamic acquisition scan $(2 \times 15$ second frames, 3 x 30 second frames, 3 x 60 second frames, 5 x 180 second frames and $4 \times 300$ second frames).

Patients were then allowed to move around freely for 40 minutes before commencing the F-18-FDG study.

\section{F-18-FDG}

A 10-minute transmission scan was acquired for attenuation correction over the $10 \mathrm{~cm}$ bed previously identified. An intravenous bolus of F-18-FDG (185 MBq; mean $177 \mathrm{MBq}$, range $106-238 \mathrm{MBq}$ ) was used to keep the cumulative effective dose low (in line with European recommendations for injected F-18-FDG activity [15]). Imaging was immediately commenced for 60 minutes for a dynamic acquisition scan ( $8 \times 15$ second frames, $4 \times 30$ second frames, $1 \times 60$ second frame, 1 x 300 second frame and 5 x 600 second frames).

\section{Quantification}

$\mathrm{SUV}_{\mathrm{BSA}}$ analysis was performed on all PET scans. A static image containing the data for the last 10 minutes of the dynamic acquisition was used to define the boundaries of the visible tumor. A standardised uptake value, scaled by body surface area $\left(\mathrm{SUV}_{\mathrm{BSA}}\right)$, was obtained from this final frame of data.

Regions of Interest (ROI) were drawn around the visible tumor in multiple slices obtained from the static image using a tumor threshold of $42 \%$ of the maximum-pixel-value 
within the tumor. The maximum $\mathrm{SUV}_{\mathrm{BSA}}$ was utilized.

\section{Imaging}

Staging CT and EUS were performed within two weeks of baseline PET. Staging was performed as per criteria laid out by the American Joint Committee on Cancer [16], and blinded to pathological staging.

\section{Surgery}

Surgery was performed three to four weeks following completion of neo-adjuvant chemotherapy.

\section{Pathology}

Surgical specimens were fixed in formaldehyde and assessed for pathological stage [16] and Tumor Regression Grade (TRG), using the Mandard criteria [17]. A pathological response was defined as a TRG of 1-3, whilst a TRG score of 4-5 indicated little or no response. The resection was assessed for completeness of resection (R0, complete tumor resection; R1, positive microscopic tumor margin).

\section{Ki-67 immunohistochemical analysis}

Ki-67 immunohistochemical analysis was performed on formalin fixed, paraffin embedded tumor tissue taken at the time of diagnostic endoscopy and surgical resection specimen. Paraffin embedded sections 3 - $4 \mu \mathrm{m}$ thick were taken from each tumor sample and de-waxed and rehydrated prior to antigen retrieval. Each section was labelled using the monoclonal mouse antibody Ki-67, according to manufacturers' protocol (clone MIB-1, DakoCytomation, Cambridgeshire, UK) at 1:500 dilution following antigen retrieval by microwaving the sections (20 minutes in microwave at 800 watts) in citrate buffer $(0.01 \mathrm{M}, \mathrm{pH} 6$, in house solution). Antibody to antigen binding was detected using the avidin-biotin complex method (as per Dako protocol, DakoCytomation, Cambridgeshire, UK). Immunohistochemistry was performed on an automated stainer (TechMate, DakoCytomation, Cambridgeshire, UK). Each run included positive and negative controls. For positive controls sections of human reactive lymph node were used with a high mitotic/proliferative rate. Primary antibodies were omitted on sections used as negative controls.

The number of ki-67 positive tumor nuclei were counted in two to five (dependent on amount of tissue available) high power fields (x40) for each sample of tumor with the proliferative activity expressed as the mean number of positively stained tumor nuclei in all fields assessed ( \pm standard deviation) as per Breeuwsma et al [18]. All of the fields examined represented tumor with no normal tissue within those fields. The pathologist was blinded to the PET results.

\section{Survival}

All patients were followed up for a minimum of one year or until death.

\section{Statistics}

Spearman Rank correlation was performed to assess the relationship between pathological response, ki-67 proliferative activity and initial tumor tracer uptake as well as percentage change in tumor uptake of each PET tracer at seven and 14 days into the first cycle of neoadjuvant chemotherapy.

Influence of pathological response, resection status, initial and percentage change in PET tracer uptake on survival was assessed using Kaplan Meier survival curves (log rank statistic).

Analysis was performed on the Statistical Package for Social Sciences (Statistical Package for the Social Sciences V13.1, Chicago, USA). A p-value of less than 0.05 denotes significance.

\section{Results}

Eighteen patients, 12 male and six female, with a mean age of 60.9 years (range 50.1 - 78.1) were recruited. The majority of cancers were adenocarcinomas $(16 / 18,89 \% ; 2 / 18,11 \%$ were squamous cell) and were esophageal in origin $(10 / 18$, $56 \% ; 8 / 18,44 \%$ were OGJ in origin). Most patients presented with stage III and IV disease $(12 / 18,67 \%)$, Table 1 . The mean blood glucose, prior to each PET scan was $5.6 \mathrm{mmol} / \mathrm{l}$ (range 4.4 - $7.7 \mathrm{mmol} / \mathrm{l}$ ).

Of 18 patients recruited into this study, 14 initially underwent neoadjuvant chemotherapy with only nine of these patients proceeding to surgery (Table 2). Two patients, upon completion of neoadjuvant chemotherapy refused surgery and underwent chemoradiotherapy instead (patients six and seven). Three patients had progression of disease during neoadjuvant chemotherapy and after discussion at the multi-disciplinary-team-meeting, surgery was substituted with chemoradiotherapy (patients 13, 17 and 18). Of four patients receiving palliative chemotherapy, only two completed the full course (patients nine and 14). Of the remaining two, one had clinical progression of disease (patient one) and underwent stenting after completing only three cycles of chemotherapy whilst the second patient died from an aortoesophageal fistula prior to chemotherapy (patient eight).

\section{PET imaging}

All 18 patients underwent baseline PET imaging with both F18-FDG and C-11-Choline. Two patients underwent no further PET imaging following baseline PET due to one patient (patient six) suffering toxic side effects of chemotherapy and 


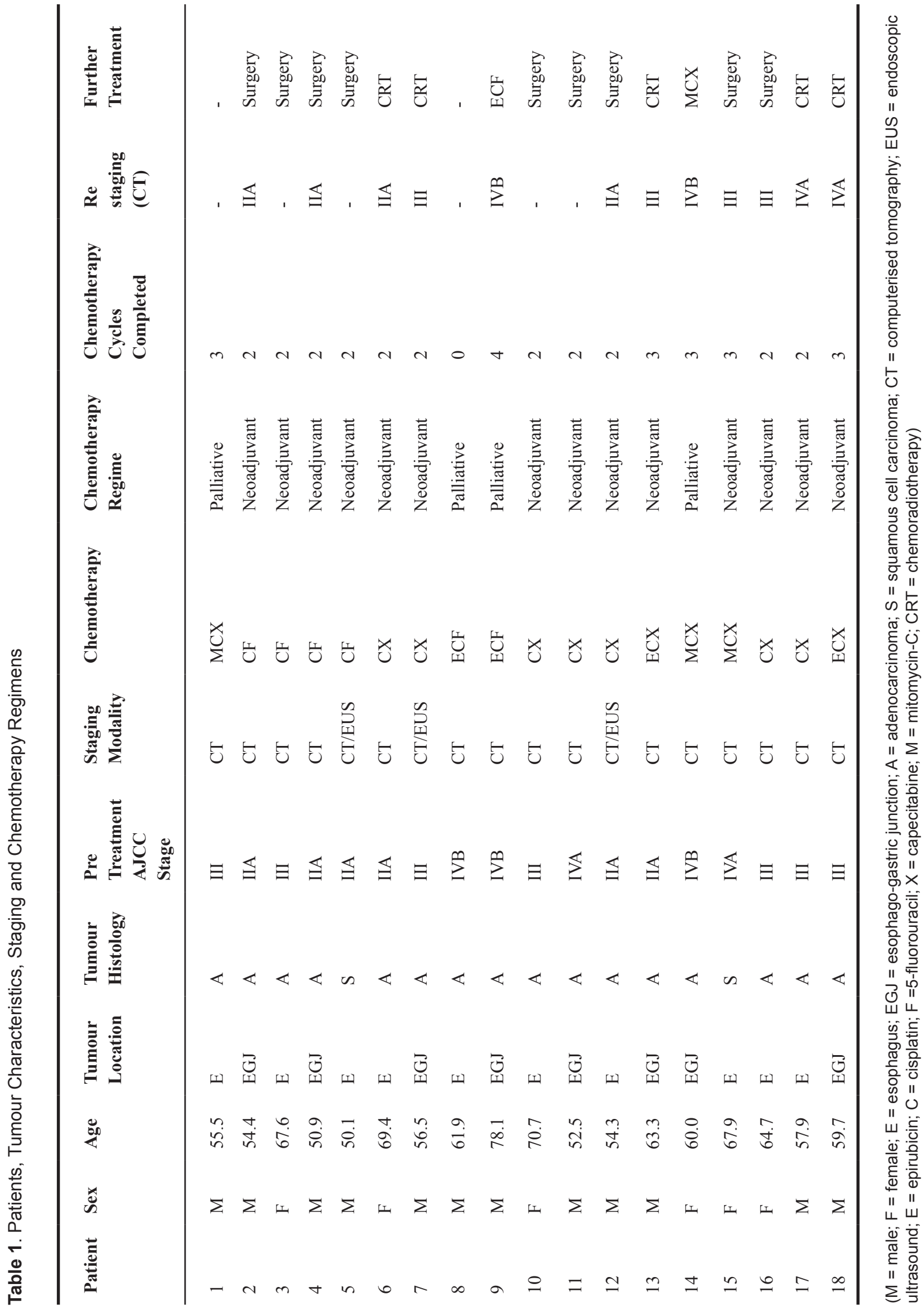




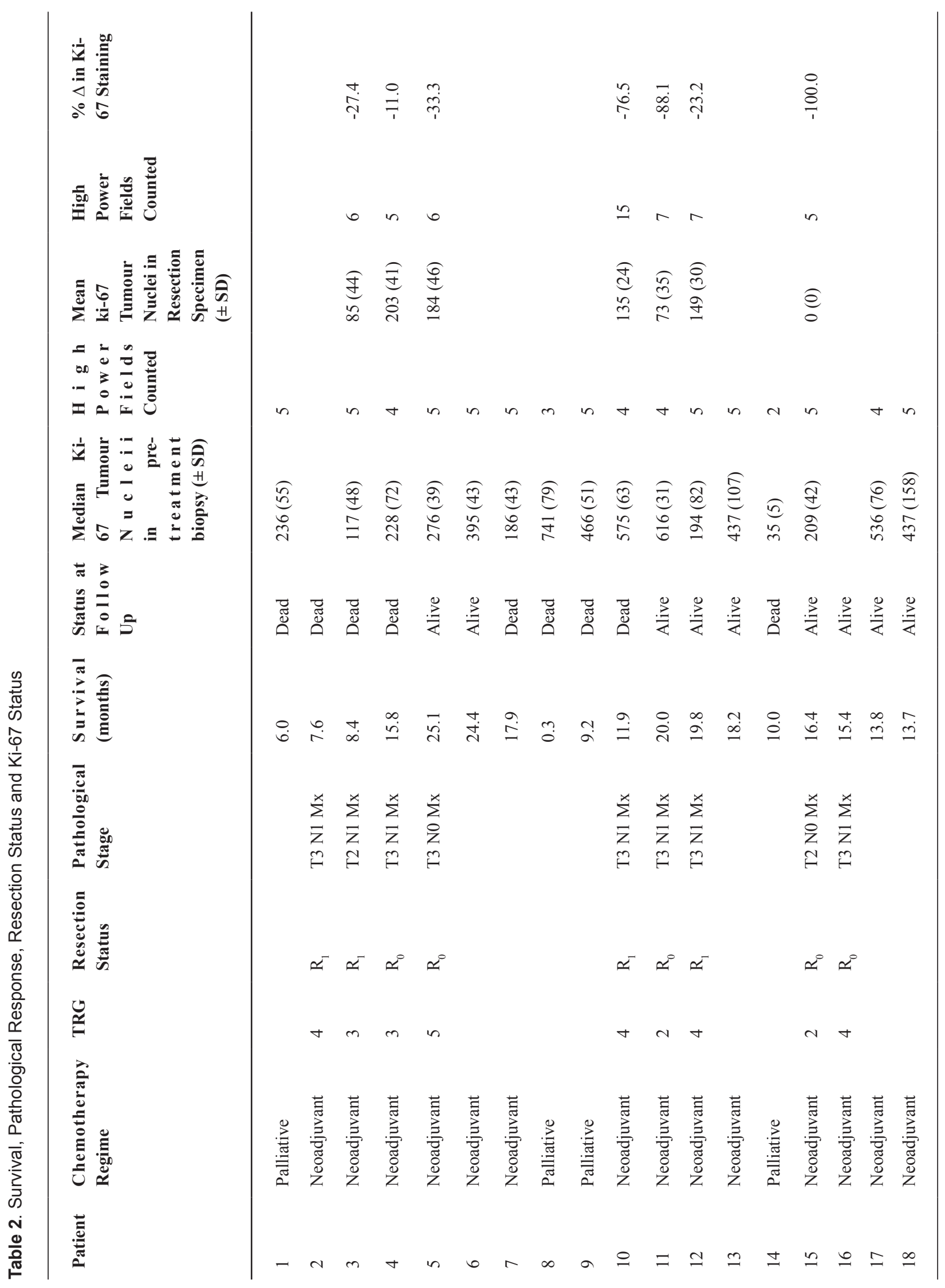




\section{C-11 Choline $\quad$ F-18 FDG}
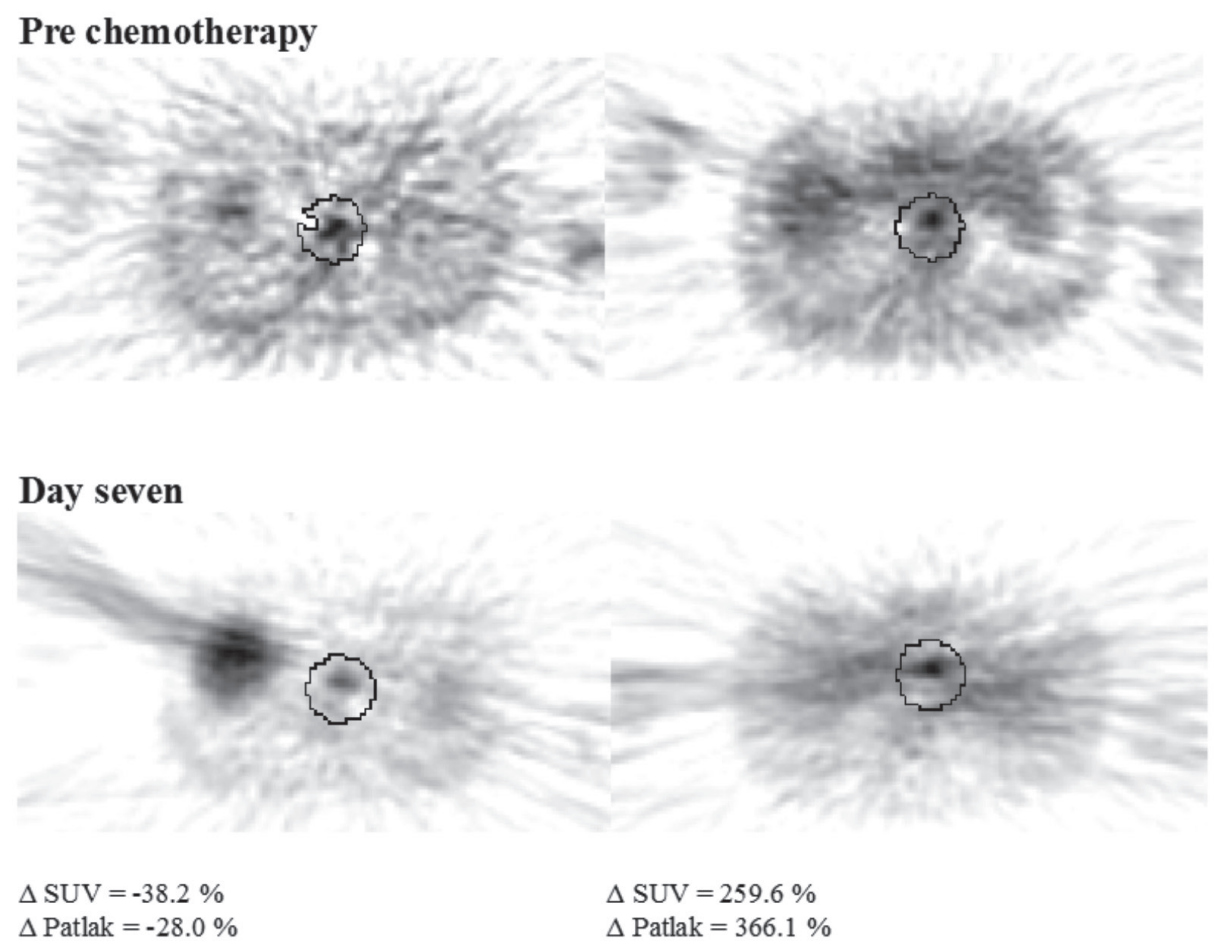

\section{Day 14}

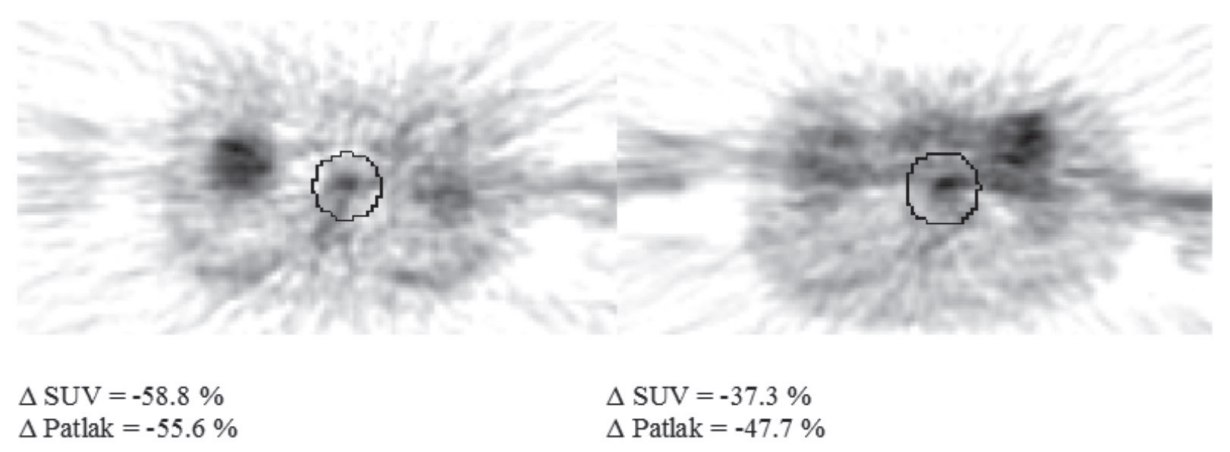

Figure 1. Trans-axial PET images of patient three, a pathological responder.

therefore unable to travel for PET imaging and one patient (patient eight) who died prior to chemotherapy. At day 14, patient 12 underwent imaging with F-18-FDG alone (problems with C-11-Choline synthesis). F-18-FDG PET detected more extensive local disease than seen on staging CT and endoscopy in patient 18 , which was confirmed on re-staging $\mathrm{CT}$ scan. There was no $\mathrm{C}$-11-Choline tumor uptake prior to chemotherapy in patients 11 and 18 .
All 18 patients (100\%) had their primary tumor imaged with F-18-FDG in contrast to just $16(89 \%)$ primary tumors successfully imaged with C-11-Choline. The two primary tumors not successfully imaged with C-11-Choline (patient 11 and 18) were sited at the esophago-gastric junction, with a high uptake of C-11-Choline within the liver obscuring the primary tumor. Neither C-11-Choline nor F-18-FDG could differentiate between primary tumors and involved peri- 


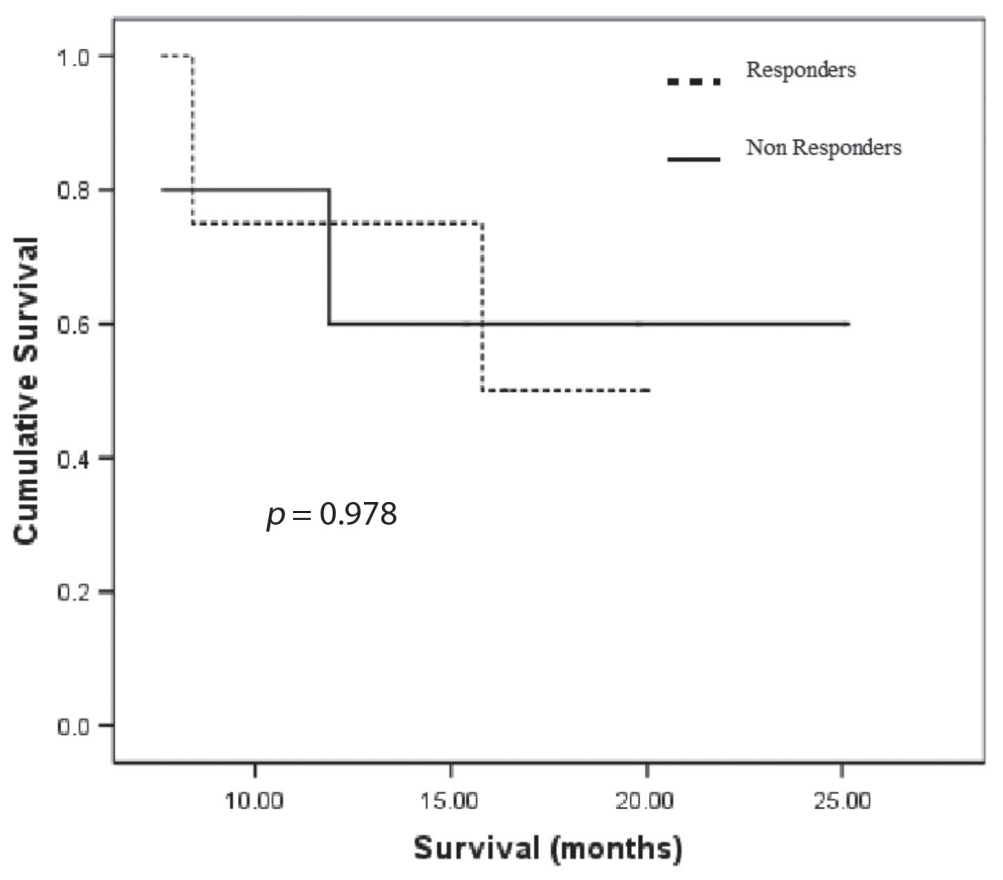

Figure 2. Kaplan Meier survival curves for pathological response.

esophageal lymph nodes.

\section{Tumor uptake}

There was no correlation between initial F-18-FDG and C11-Choline uptake $(r=-0.07, p=0.80)$ with no significant difference between mean initial uptake for F-18-FDG (0.19) and C-11-Choline (0.18), $\mathrm{p}=0.88$.

There was no significant correlation of percentage change in tracer uptake at day seven (F-18-FDG vs C-11Choline, $r=-0.33, p=0.33)$ and at day $14(\mathrm{r}=-0.33, \mathrm{p}=$ $0.38)$.

\section{Pathological response}

Nine of 14 patients initially receiving neoadjuvant chemotherapy proceeded to surgery. Four of these nine (44\%) had a pathological response (TRG 2-3), with five patients undergoing a curative (R0) resection (Table 2). Figure 1 displays images obtained with each PET tracer.

There was no significant correlation of the initial tumor tracer uptake for either F-18-FDG $(n=9, r=-0.17, p=0.66)$ or C-11-Choline $(\mathrm{n}=8, \mathrm{r}=0.51, \mathrm{p}=0.19)$ with pathological response. There was no significant correlation between percentage change in uptake of either tracer and pathological response or TRG at day seven or 14, although percentage change in C-11-Choline uptake at day 14 and TRG showed a trend towards significance $(\mathrm{r}=0.95, \mathrm{p}=0.05)$.

\section{Pathological response, resection status and survival}

Follow-up ranged from 0.3 - 25.1 months. There was no significant difference in median survival between pathological responders ( $\mathrm{n}=4,16.1$ months) and non-responders $(\mathrm{n}=$ 5, 19.0 months), $p=0.98$ (Fig. 2). Resection status had no significant impact on survival $\left(\mathrm{R}_{0}: \mathrm{n}=5,22.8\right.$ months; $\mathrm{R}_{1}: \mathrm{n}$ $=4,11.9$ months $), p=0.07$.

For survival analysis the patients were dichotomised based on the median initial tumor PET tracer uptake (i.e. greater or less than the median tumor PET tracer uptake) or on median percentage change in uptake (i.e. greater or less than the median $\% \Delta$ tumor PET tracer uptake). There was no significant difference in survival between the two groups (Table 3a, b).

\section{Ki-67 staining}

Ki-67 immunohistochemical staining was performed in 16 out 18 patients on the pre treatment endoscopic biopsy (Table 2). All pre treatment tumor tissue showed positive ki-67 nuclear staining. All patients receiving neo-adjuvant chemotherapy had a reduction ki-67 nuclear staining (Table 2).

There was no significant correlation of the mean number of ki-67 positive tumor nuclei in pre treatment tumor samples with initial tumor uptake of either F-18-FDG or C11-Choline, (C-11-Choline: $r=-0.17, p=0.57, n=14$; F-18FDG: $r=0.24, p=0.37, n=16$ ). There was no significant 
Table 3a. Median Initial Tumor PET Tracer Uptake and Survival

\begin{tabular}{|c|c|c|c|c|c|}
\hline Quantification & Tracer & $\begin{array}{l}\text { Median } \\
\text { Uptake } \\
( \pm \text { SD) }\end{array}$ & $\begin{array}{l}\text { Survival, months } \\
( \pm \text { SD })\end{array}$ & $\mathbf{p}$ & $\mathbf{n}$ \\
\hline \multirow[t]{2}{*}{ SUV } & F-18 FDG & $0.17(0.06)$ & $\begin{array}{l}<\text { median } 14.8(2.0) \\
>\text { median } 17.2(2.7\end{array}$ & 0.70 & 18 \\
\hline & C-11 Choline & $0.15(0.01)$ & $\begin{array}{l}<\text { median } 19.3(2.7) \\
>\text { median } 11.5(2.3)\end{array}$ & 0.11 & 16 \\
\hline
\end{tabular}

correlation of change in ki-67 tumor staining with change in tumor tracer uptake over time (F-18-FDG day $7: \mathrm{r}=0.31, \mathrm{p}$ $=0.54, \mathrm{n}=6$; day 14: $\mathrm{r}=-0.40, \mathrm{p}=0.51, \mathrm{n}=5$ : C-11-Choline day $7: r=-0.60, p=0.29, n=5$; day $14: r=-0.50, p=0.67$, $\mathrm{n}=3$ ).

There was no significant correlation of the pre-treatment tumor ki-67 staining with pathological response $(\mathrm{r}=-0.14, \mathrm{p}$ $=0.76, \mathrm{n}=7$ ) and percentage change in tumor ki-67 staining and pathological response $(\mathrm{r}=-0.14, \mathrm{p}=0.76, \mathrm{n}=7)$.

For survival analysis the patients were dichotomised based on the median number of tumor nuclei staining positive for ki-67 in pre treatment samples. The median number of ki-67 positive tumor nuclei was 336 per high power field per sample. There was no significant difference in survival (more than median ki-67 staining: 17.9 months $\pm-3.1, \mathrm{n}=8$; less than median: 15.8 months $\pm 2.6, \mathrm{n}=8), \mathrm{p}=0.51$.

The median change in ki-67 expression following neoadjuvant chemotherapy was $-33.3 \%$ ( $\mathrm{SD} \pm 35.8)$. For survival analysis, the patients were divided into two groups based on median change in ki-67 histochemical expression. There was no significant difference in the median survival between those patients with a greater than median reduction in ki-67 staining (14.7 months $\pm 2.7, \mathrm{n}=5)$ and those with a lesser reduction in ki-67 expression (21.8 months $\pm 2.9, \mathrm{n}=3), \mathrm{p}$ $=0.32$.

\section{Discussion}

The majority of studies utilizing PET to monitor response to treatment in esophago-gastric cancers have employed F18-FDG with no studies using C-11-Choline. C-11-Choline has only been used in two studies for imaging of esophagogastric cancers $[19,20]$.

\section{Imaging of tumor}

In this study F-18-FDG successfully imaged all 18 primary tumors, irrespective of tumor location and histological type. C-11-Choline was unable to image two primaries, both substantial adenocarcinomas at the OGJ. This limitation of C11-Choline PET is related to the considerable non specific uptake of C-11-Choline within the liver [19, 20]. A further hypothesis for this was proposed by Jager et al [19], stating

Table 3b. Median \% $\Delta$ in SUV PET Tumour Tracer Uptake at Seven and 14 days into the First Cycle of Chemotherapy and Survival

\begin{tabular}{|c|c|c|c|c|c|}
\hline PET Timing & Tracer & $\begin{array}{l}\text { Median \% } \% \text { SUV } \\
( \pm \text { SD) }\end{array}$ & Survival, months $( \pm$ SD) & $\mathbf{p}$ & n \\
\hline \multirow[t]{2}{*}{ Day 7} & F-18 FDG & $-3.3(81.9)$ & $\begin{array}{l}<\text { Median } 15.0(2.3) \\
>\text { Median } 20.7(2.7)\end{array}$ & 0.43 & 13 \\
\hline & C-11 Choline & $0.0(24.1)$ & $\begin{array}{l}<\text { Median } 21.3(3.4) \\
>\text { Median } 14.1(2.1)\end{array}$ & 0.24 & 11 \\
\hline \multirow[t]{2}{*}{ Day 14} & F-18 FDG & $-17.1(28.3)$ & $\begin{array}{l}<\text { Median } 18.1(1.2) \\
>\text { Median } 17.2(3.3)\end{array}$ & 0.41 & 12 \\
\hline & C-11 Choline & $-0.2(36.9)$ & $\begin{array}{l}<\text { Median } 21.1(3.4) \\
>\text { Median } 13.2(2.1)\end{array}$ & 0.16 & 9 \\
\hline
\end{tabular}


that primary tumors had a considerable lower SUV uptake of C-11-Choline thereby causing difficulty in distinguishing tumor from normal tissue. This finding may be due to differing methods of quantification, with our study showing no such difference in uptake. Kobori et al [20] found C-11-Choline to be more sensitive in identifying small tumors than F-18FDG, whilst Jager et al found that irrespective of tumor size, F-18-FDG was superior at imaging of the primary tumor. In our study, neither tracer was able to distinguish primary tumor from peri-esophageal disease, in keeping with previous reports [19].

\section{Pathological response}

A pathological response was achieved in $44 \%$ of patients in this study, consistent with population based data [21]. This study found no significant correlation of pre-treatment tumor uptake of C-11-Choline or F-18-FDG with pathological response, in keeping with previous reports $[22,23]$. We found, as did others $[22,24]$, that change in uptake of either tracer at each time point could not significantly distinguish between each TRG. The percentage change in C-11-Choline at day 14 strongly correlated with TRG $(\mathrm{r}=0.95, \mathrm{p}=0.05)$, although there were few responders in this group. When TRG are amalgamated into groups indicating either responders or non responders, the change in C-11-Choline uptake appears to have some success in distinguishing between them. Only five studies have assessed F-18-FDG and pathological response during the course of chemotherapy [6] or chemoradiotherapy $[2,23,25,26]$. Wieder et al performed F-18-FDG PET after two weeks and upon completion of chemoradiotherapy for squamous cell carcinoma of the esophagus [23]. Using ROC analysis, a percentage change in SUV uptake of $30 \%$ (at two weeks) was able to distinguish responders from non responders. Kroep et al, using F-18-FDG PET after the second of six cycles of chemotherapy for both adenocarcinoma and squamous cell carcinoma, reported a similar finding, although they identified the threshold change in SUV for predicting response at 40\% [6]. Using F-18-FDG PET to predict pathological response earlier than two weeks into the treatment regime appears to be too early in order to distinguish responders from non responders [25]. A recent study looking at change in F-18-FDG uptake during and upon completion of therapy found no correlation between tracer uptake and pathological response [26]. Studies identifying a link between change in F-18-FDG uptake and pathological response, did so with a high sensitivity and low specificity (range 55-86 \%) for identifying responders [6, 23, 27] (i.e. up to $45 \%$ of non-responders would be classified as responders according to F-18-FDG PET). Studies have shown that patients with a poor response to neoadjuvant therapy have a worse prognosis than those who did not have neoadjuvant therapy [28]. It is these non responders whom we need to identify, accurately and at an early stage so that treatment can be altered.

\section{Survival}

We found that pathological response did not predict one year survival, although the analysis only included nine patients. Wieder and Brucher et al found that change in F-18-FDG correlated with pathological response, which in turn conferred a survival advantage $[23,27]$. In a study designed to evaluate the impact of pathological response, Dunne et al reported that pathological response was not an independent prognostic indicator [29], a finding reiterated by others [30]. A limitation of assessing pathological response, is that subjective criteria, open to sampling errors are used. We found that at day 14 into the first cycle of chemotherapy, patients with a greater reduction in C-11-Choline, had a worse prognosis than those with a smaller reduction. This phenomenon may relate to tumors with a high proliferation rate are more responsive to therapy $[31,32]$. The only factors strongly influencing survival in our study were the presence of a curative resection and the change in $\mathrm{C}$-11-Choline uptake at day 14.

\section{Cellular proliferation}

All pre-treatment tumors were positive for ki-67, with all who received neoadjuvant chemotherapy having a reduction in ki-67 staining. Choline and its metabolites have been found in increased levels in a variety of tumors [33, 34]. Cell populations with a high proliferative activity are known to have higher rates of choline transport into cells compared with slower growing populations $[35,36]$ suggesting a correlation between choline uptake and proliferation [37-42]. Several studies reported that F-18-FDG uptake correlates with viable cell number rather than proliferation [43-46], although recent studies have reported a correlation between ki-67 staining and F-18-FDG uptake in lung, brain and ovarian tumors [47-50], but not in esophageal cancers [45]. This study found no correlation of ki-67 staining with initial C11-Choline or F-18-FDG tumor uptake. This absence of correlation with pre-therapy tumors may be due to the heterogeneous nature of tumors even within a single tumor type. In some cases where we obtained multiple samples from single tumors we observed a wide variation in ki-67 protein expression. In contrast the PET tracer incorporation was determined on the whole tumor. Two studies assessing correlation of C-11-Choline uptake with ki-67 proliferative index in brain and prostate tumors came to opposite conclusions [18, 40]. Breeuwsma et al found that C-11-Choline uptake within prostate cancer showed no significant correlation with ki-67 [18], whilst Utriainen et al reported a strong correlation of ki67 proliferative index with $\mathrm{C}$-11-Choline uptake [40]. Using proton magnetic resonance spectroscopy they found that the concentration of choline containing compounds within the 
tumors investigated correlated with the ki-67 proliferation index although there was no correlation of the concentration of the choline compounds with C-11-Choline uptake [40].

\section{Limitations}

In keeping with the majority of PET studies, the major limitation was patient numbers. Only recently has an adequately powered F-18-FDG PET study been performed, utilizing F18 -FDG to predict response to neoadjuvant chemotherapy as well as influence treatment [51] in OGJ cancers. The patients were a heterogeneous group, in terms of chemotherapy (which may have an effect on response as well as tumor tracer uptake [52]), tumor types and stage, all of which may have had an impact.

F-18-FDG PET is superior for imaging of esophageal and OGJ cancers. Neither tracer was able to predict pathological response or survival. C-11-Choline uptake did not directly relate to cellular proliferation.

\section{Acknowledgment}

Chief Scientists Office (CSO CZG/1/134). The Scottish Hospital Endowments Research Trust (SHERT), Mrs Robina Menzies Smith, Medical Research Fellowship. The sponsors had no input on the study design, in the collection, analysis and interpretation of data; in the writing of the manuscript; and in the decision to submit the manuscript for publication.

\section{References}

1. Spaepen K, Stroobants S, Dupont P, Bormans G, Balzarini J, Verhoef G, Mortelmans L, et al. [(18)F]FDG PET monitoring of tumor response to chemotherapy: does [(18)F]FDG uptake correlate with the viable tumor cell fraction? Eur J Nucl Med Mol Imaging 2003;30(5):682688.

2. Wieder HA, Beer AJ, Lordick F, Ott K, Fischer M, Rummeny EJ, Ziegler S, et al. Comparison of changes in tumor metabolic activity and tumor size during chemotherapy of adenocarcinomas of the esophagogastric junction. J Nucl Med 2005;46(12):2029-2034.

3. Jamieson GG, Mathew G, Ludemann R, Wayman J, Myers JC, Devitt PG. Postoperative mortality following esophagectomy and problems in reporting its rate. $\mathrm{Br} \mathrm{J}$ Surg 2004;91(8):943-947.

4. McAteer D, Wallis F, Couper G, Norton M, Welch A, Bruce D, Park K, et al. Evaluation of 18F-FDG positron emission tomography in gastric and esophageal carcinoma. Br J Radiol 1999;72(858):525-529.

5. Couper GW, McAteer D, Wallis F, Norton M, Welch A, Nicolson M, Park KG. Detection of response to chemotherapy using positron emission tomography in patients with esophageal and gastric cancer. Br J Surg 1998;85(10):1403-1406.

6. Kroep JR, Van Groeningen CJ, Cuesta MA, Craanen ME, Hoekstra OS, Comans EF, Bloemena E, et al. Positron emission tomography using 2-deoxy-2-[18F]-fluoro-Dglucose for response monitoring in locally advanced gastroesophageal cancer; a comparison of different analytical methods. Mol Imaging Biol 2003;5(5):337-346.

7. Weber WA, Ott K, Becker K, Dittler HJ, Helmberger H, Avril NE, Meisetschlager G, et al. Prediction of response to preoperative chemotherapy in adenocarcinomas of the esophagogastric junction by metabolic imaging. J Clin Oncol 2001;19(12):3058-3065.

8. Zeisel SH. Dietary choline: biochemistry, physiology, and pharmacology. Annu Rev Nutr 1981;1(95-121.

9. Zeisel SH. Choline: an essential nutrient for humans. Nutrition 2000;16(7-8):669-671.

10. Park K. COGNATE: Cancer of the esophagus or gastricus: new assessment of the technology of endosonography. http://www.bangor.ac.uk/imscar/cognate/ 2004.

11. Siewert JR, Stein HJ. Classification of adenocarcinoma of the esophagogastric junction. Br J Surg 1998;85(11):1457-1459.

12. MRC Esophageal Cancer Working Party. Surgical resection with or without preoperative chemotherapy in esophageal cancer: a randomised controlled trial. Lancet 2002;359(9319):1727-1733.

13. Cunningham D, Starling N, Rao S, Iveson T, Nicolson M, Coxon F, Middleton G, et al. Capecitabine and oxaliplatin for advanced esophagogastric cancer. N Engl J Med 2008;358(1):36-46.

14. Du Bois D, Du Bois EF. A formula to estimate the approximate surface area if height and weight be known. Archives of Internal Medicine 1916;17:863-871.

15. Bombardieri E, Aktolun C, Baum RP, Bishof-Delaloye A, Buscombe J, Chatal JF, Maffioli L, et al. FDG-PET: procedure guidelines for tumor imaging. Eur $\mathrm{J}$ Nucl Med Mol Imaging 2003;30(12):BP115-124.

16. AJCC. Esophagus. 6th ed. New York: Springer, 2002: 91-98.

17. Mandard AM, Dalibard F, Mandard JC, Marnay J, Henry-Amar M, Petiot JF, Roussel A, et al. Pathologic assessment of tumor regression after preoperative chemoradiotherapy of esophageal carcinoma. Clinicopathologic correlations. Cancer 1994;73(11):2680-2686.

18. Breeuwsma AJ, Pruim J, Jongen MM, Suurmeijer AJ, Vaalburg W, Nijman RJ, de Jong IJ. In vivo uptake of $[11 \mathrm{C}]$ choline does not correlate with cell proliferation in human prostate cancer. Eur J Nucl Med Mol Imaging 2005;32(6):668-673.

19. Jager PL, Que TH, Vaalburg W, Pruim J, Elsinga P, Plukker JT. Carbon-11 choline or FDG-PET for staging of esophageal cancer? Eur J Nucl Med 2001;28(12):1845- 
1849.

20. Kobori O, Kirihara Y, Kosaka N, Hara T. Positron emission tomography of esophageal carcinoma using (11)C-choline and (18)F-fluorodeoxyglucose: a novel method of preoperative lymph node staging. Cancer 1999;86(9):1638-1648.

21. Gilbert FJ, Park K, Thompson AM. Scottish Audit of Gastric and Esophageal Cancer: Report 1997-2000 - A prospective audit http://www.show.scot.nhs.uk/crag/ 2002.

22. Swisher SG, Erasmus J, Maish M, Correa AM, Macapinlac H, Ajani JA, Cox JD, et al. 2-Fluoro-2-deoxyD-glucose positron emission tomography imaging is predictive of pathologic response and survival after preoperative chemoradiation in patients with esophageal carcinoma. Cancer 2004a;101(8):1776-1785.

23. Wieder HA, Brucher BL, Zimmermann F, Becker K, Lordick F, Beer A, Schwaiger M, et al. Time course of tumor metabolic activity during chemoradiotherapy of esophageal squamous cell carcinoma and response to treatment. J Clin Oncol 2004;22(5):900-908.

24. Melcher L, Wong W, Sanghera B, Bentzen SM, Hall M, Chambers J, Townsend E, et al. Sequential FDG-PET scanning in the assessment of response to neoadjuvant chemotherapy in operable esophageal cancer. J Clin Oncol 2004;22(14S):4056.

25. Gillham CM, Lucey JA, Keogan M, Duffy GJ, Malik V, Raouf AA, O’Byrne K, et al. (18)FDG uptake during induction chemoradiation for esophageal cancer fails to predict histomorphological tumor response. Br J Cancer 2006;95(9):1174-1179.

26. Smithers BM, Couper GC, Thomas JM, Wong D, Gotley DC, Martin I, Harvey JA, et al. Positron emission tomography and pathological evidence of response to neoadjuvant therapy in adenocarcinoma of the esophagus. Dis Esophagus 2008;21(2):151-158.

27. Brucher BL, Weber W, Bauer M, Fink U, Avril N, Stein HJ, Werner M, et al. Neoadjuvant therapy of esophageal squamous cell carcinoma: response evaluation by positron emission tomography. Ann Surg 2001;233(3):300309.

28. Ancona E, Ruol A, Santi S, Merigliano S, Sileni VC, Koussis H, Zaninotto G, et al. Only pathologic complete response to neoadjuvant chemotherapy improves significantly the long term survival of patients with resectable esophageal squamous cell carcinoma: final report of a randomized, controlled trial of preoperative chemotherapy versus surgery alone. Cancer 2001;91(11):21652174.

29. Dunne B, Reynolds JV, Mulligan E, Kelly A, Griffin M. A pathological study of tumor regression in esophageal adenocarcinoma treated with preoperative chemoradiotherapy. J Clin Pathol 2001;54(11):841-845.

30. Griffith JF, Chan AC, Chow LT, Leung SF, Lam YH,
Liang EY, Chung SC, et al. Assessing chemotherapy response of squamous cell esophageal carcinoma with spiral CT. Br J Radiol 1999;72(859):678-684.

31. Beardsmore DM, Verbeke CS, Davies CL, Guillou PJ, Clark GW. Apoptotic and proliferative indexes in esophageal cancer: predictors of response to neoadjuvant therapy [corrected]. J Gastrointest Surg 2003;7(1):77-86; discussion 86-77.

32. Imdahl A, Bognar G, Schulte-Monting J, Schoffel U, Farthmann EH, Ihling C. Predictive factors for response to neoadjuvant therapy in patients with esophageal cancer. Eur J Cardiothorac Surg 2002;21(4):657-663.

33. Aboagye EO, Bhujwalla ZM. Malignant transformation alters membrane choline phospholipid metabolism of human mammary epithelial cells. Cancer Res 1999;59(1):80-84.

34. Bhakoo KK, Williams SR, Florian CL, Land H, Noble MD. Immortalization and transformation are associated with specific alterations in choline metabolism. Cancer Res 1996;56(20):4630-4635.

35. Al-Saeedi F, Welch AE, Smith TA. [methyl-3H]Choline incorporation into MCF7 tumor cells: correlation with proliferation. Eur J Nucl Med Mol Imaging 2005;32(6):660-667.

36. Ramirez de Molina A, Rodriguez-Gonzalez A, Gutierrez R, Martinez-Pineiro L, Sanchez J, Bonilla F, Rosell $\mathrm{R}$, et al. Overexpression of choline kinase is a frequent feature in human tumor-derived cell lines and in lung, prostate, and colorectal human cancers. Biochem Biophys Res Commun 2002;296(3):580-583.

37. Daly PF, Lyon RC, Faustino PJ, Cohen JS. Phospholipid metabolism in cancer cells monitored by 31P NMR spectroscopy. J Biol Chem 1987;262(31):14875-14878.

38. Kalra R, Wade KE, Hands L, Styles P, Camplejohn R, Greenall M, Adams GE, et al. Phosphomonoester is associated with proliferation in human breast cancer: a 31P MRS study. Br J Cancer 1993;67(5):1145-1153.

39. Smith TA, Eccles S, Box G, Titley JC, Leach MO, McCready VR. Phosphocholine and choline content of rat sarcoma cells grown in the presence and absence of serum. Anticancer Res 1996;16(3B):1389-1392.

40. Utriainen M, Komu M, Vuorinen V, Lehikoinen P, Sonninen P, Kurki T, Utriainen T, et al. Evaluation of brain tumor metabolism with [11C]choline PET and 1H-MRS. J Neurooncol 2003;62(3):329-338.

41. Smith TA, Eccles S, Ormerod MG, Tombs AJ, Titley JC, Leach MO. The phosphocholine and glycerophosphocholine content of an oestrogen-sensitive rat mammary tumor correlates strongly with growth rate. Br J Cancer 1991;64(5):821-826.

42. Yoshimoto M, Waki A, Obata A, Furukawa T, Yonekura Y, Fujibayashi Y. Radiolabeled choline as a proliferation marker: comparison with radiolabeled acetate. Nucl Med Biol 2004;31(7):859-865. 
43. Yap CS, Czernin J, Fishbein MC, Cameron RB, Schiepers C, Phelps ME, Weber WA. Evaluation of thoracic tumors with $18 \mathrm{~F}$-fluorothymidine and $18 \mathrm{~F}$-fluorodeoxyglucose-positron emission tomography. Chest 2006;129(2):393-401.

44. Abe Y, Matsuzawa T, Fujiwara T, Fukuda H, Itoh M, Yamada K, Yamaguchi K, et al. Assessment of radiotherapeutic effects on experimental tumors using 18F-2-fluoro-2-deoxy-D-glucose. Eur J Nucl Med $1986 ; 12(7): 325-328$

45. van Westreenen HL, Cobben DC, Jager PL, van Dullemen HM, Wesseling J, Elsinga PH, Plukker JT. Comparison of 18F-FLT PET and 18F-FDG PET in esophageal cancer. J Nucl Med 2005a;46(3):400-404.

46. Higashi K, Clavo AC, Wahl RL. Does FDG uptake measure proliferative activity of human cancer cells? In vitro comparison with DNA flow cytometry and tritiated thymidine uptake. J Nucl Med 1993b;34(3):414-419.

47. Higashi K, Ueda Y, Yagishita M, Arisaka Y, Sakurai A, Oguchi M, Seki H, et al. FDG PET measurement of the proliferative potential of non-small cell lung cancer. $\mathrm{J}$ Nucl Med 2000;41(1):85-92.

48. Vesselle H, Schmidt RA, Pugsley JM, Li M, Kohlmyer SG, Vallires E, Wood DE. Lung cancer prolifera- tion correlates with [F-18]fluorodeoxyglucose uptake by positron emission tomography. Clin Cancer Res 2000;6(10):3837-3844.

49. Avril N, Menzel M, Dose J, Schelling M, Weber W, Janicke F, Nathrath W, et al. Glucose metabolism of breast cancer assessed by 18F-FDG PET: histologic and immunohistochemical tissue analysis. J Nucl Med 2001;42(1):9-16.

50. Kurokawa T, Yoshida Y, Kawahara K, Tsuchida T, Okazawa H, Fujibayashi Y, Yonekura Y, et al. Expression of GLUT-1 glucose transfer, cellular proliferation activity and grade of tumor correlate with [F-18]-fluorodeoxyglucose uptake by positron emission tomography in epithelial tumors of the ovary. Int $\mathrm{J}$ Cancer 2004;109(6):926-932.

51. Lordick F, Ott K, Krause BJ, Weber WA, Becker K, Stein HJ, Lorenzen S, et al. PET to assess early metabolic response and to guide treatment of adenocarcinoma of the esophagogastric junction: the MUNICON phase II trial. Lancet Oncol 2007;8(9):797-805.

52. Suttie SA, Park KG, Smith TA. [18F]2-fluoro-2-deoxyD-glucose incorporation by AGS gastric adenocarcinoma cells in vitro during response to epirubicin, cisplatin and 5-fluorouracil. Br J Cancer 2007;97(7):902-909. 